\title{
Association between fetal-type posterior cerebral artery and intracranial anterior and posterior circulating atherosclerotic plaque on high-resolution MRI at 3.0T
}

\author{
Dingqi Liu \\ Zhongda Hospital Southeast University

\section{Ruijing Xin} \\ Nanjing Drum Tower Hospital \\ Deng-Ling Zhao \\ Zhongda Hospital Southeast University \\ Xiao-Hui Chen \\ Zhongda Hospital Southeast University \\ Cheng Li ( $\square$ cjr.licheng@vip.163.com ) \\ Zhongda Hospital Southeast University
}

\section{Research Article}

Keywords: Atherosclerosis, posterior cerebral artery (PCA), plaque, circle of Willis, high-resolution MRI

Posted Date: March 1st, 2022

DOl: https://doi.org/10.21203/rs.3.rs-1373363/v1

License: (c) (1) This work is licensed under a Creative Commons Attribution 4.0 International License.

Read Full License 


\section{Abstract \\ Objectives}

Atherosclerotic high-risk plaque rupture is one of the important factors leading to ischemic stroke. The present study used HRMRI to quantitatively analyze the association between the fetal-type posterior artery and intracranial anterior and posterior circulating atherosclerotic plaques.

\section{Materials and Methods}

A total of 58 patients ( 25 patients with fetal-type posterior artery and 33 patients without fetal-type posterior artery) were included for 3.0T HRMRI scan analysis. The images of the MCA and VBA vessel wall on HRMRI were compared between the two groups based on the degree of stenosis, remodeling index, and eccentricity degree.

\section{Results}

Positive remodeling of MCA plaques was more frequently observed in the fetal-type posterior artery group than in the control group $(P<0.05)$. The remodeling Index $(95 \% \mathrm{Cl}, 0.001-0.474 ; \mathrm{P}=0.015)$ was associated with fetal-type posterior artery following the adjustment for the confounding factors. No significant differences were found in the VBA plaque characteristics between the two groups $(P>0.05)$.

\section{Conclusion}

Fetal-type posterior artery promotes the development of atherosclerosis plaque and positive vessel remodeling in middle cerebral artery, whereas it exhibits no association with vertebrobasilar artery plaque formation.

\section{Introduction}

Stroke has a high incidence, disability, and mortality rate, and has become one of the most important diseases endangering human health [1]. Ischemic stroke is one of the various stroke types, which accounts for $75-90 \%$ of all strokes. The pathogenesis of ischemic stroke includes artery-to-artery embolism, hemodynamic damage, and occlusion of penetrating arteries [2]. Artery-to-artery embolism is the main cause of disease. The main source of emboli in ischemic stroke is the atherosclerotic high-risk plaque rupture. Therefore, it is important to identify the presence of high-risk plaques at an earlier stage $[3,4]$.

Collaterals are considered to be a predisposition factor for atherosclerotic progression and cerebral infarction [5]. Well-developed collateral circulation plays a significant role in maintaining cerebral blood 
flow. The circle of Willis and the leptomeningeal collaterals are the primary and secondary collateral circulation structures in the brain. The circle of Willis is a communication artery ring of the anterior and posterior circulation. However, only $42-52 \%$ of healthy subjects exhibit a configuration of the circle of Willis $[6,7]$. Fetal-type posterior artery (FTP) is a common structural incomplete variation of the circle of Willis. FTP directly originates from the internal carotid artery and complete FTP (cFTP) is characterized by the absence of the P1 segment, while partial FTP (pFTP) is characterized by hypoplastic of P1 segment. According to previous studies, it is generally suggested that FTP is present in approximately 4$46 \%$ of the worldwide population $[8,9]$.

At present, a high number of studies have examined the collateral circulation in the FTP and its association with the incidence of ischemic stroke. However, only a limited number of studies have assessed the difference between anterior and posterior cerebral circulating artery plaques caused by FTP. The present study aimed to use HRMRI to quantitatively analyze the association between FTP and the plaques in the intracranial vessels.

\section{Methods}

\section{Study sample}

Patients who had undergone HRMRI examinations in one affiliated University hospital from October 2018 to October 2021 were retrospectively recruited. The inclusion criteria were the following: (1) Age $\geq 18$ years; (2) the plaques on HRMRI were decided by neuroradiologists. The exclusion criteria were the following: (1) Intracranial segment of internal carotid artery stenosis $>50 \%$; (2) vasculopathy, including dissection, vasculitis, or moyamoya disease; (3) cardioembolism and hemorrhagic stroke. The study protocol was approved by the Institutional review board and all subjects provided written informed consent forms for their participation in the study. All methods were performed in accordance with Declaration of Helsinki.

\section{Imaging protocol}

MRI was performed with a 3.0 T MRI scanner (Philips Ingenia II) and a sixteen-channel phased array coil. All patients underwent 3D-TOF, conventional brain MRI scans (DWI, ADC, T1WI, T2WI, FLAIR), and 3D T1WI HRMRI. HRMRI was performed using non-contrast and postcontrast 3D T1WI-VISTA sequence and 3D time-of-flight (TOF) MRA sequence. Before acquisition of the contrast-enhanced 3D T1WI-VISTA sequence, $0.1 \mathrm{ml} / \mathrm{kg}$ of gadolinium contrast agent $(0.1 \mathrm{mg} / \mathrm{ml}$; Magnevist; Schering, Berlin, Germany) was administered to the patient.

3D-TOF MRA was obtained in the axial plane and the scanning parameters were as follows: Repetition time $/$ echo time $(T R / T E)=15 \mathrm{~ms} / 3.5 \mathrm{~ms}$; flip angle $=18^{\circ}$; slice thickness $=0.5 \mathrm{~mm}$; matrix size $=268 \mathrm{~mm} \times$ $180 \mathrm{~mm}$; field of view $(\mathrm{FOV})=200 \mathrm{~mm} \times 200 \mathrm{~mm}$; Voxel $=0.75 \times 0.75 \times 0.75 ;$ number of average $(\mathrm{NEX})=1$; TOF-MRA scan time of 4 min. 
Non-contrast and postcontrast HRMRI was obtained on the coronal plane. The three-dimensional turbo spin echo sequence (3D VISTA sequence) was used to inhibit the blood flow and optimize the display of the intracranial vascular wall. The scanning parameters were as follows: Repetition time/echo time $(\mathrm{TR} / \mathrm{TE})=800 \mathrm{~ms} / 16 \mathrm{~ms}$; flip angle $=90^{\circ}$; slice thickness $=0.5 \mathrm{~mm}$; matrix size $=400 \mathrm{~mm} \times 400 \mathrm{~mm}$; field of view $($ FOV $)=200 \mathrm{~mm} \times 200 \mathrm{~mm}$; Voxel $=0.5 \times 0.5 \times 0.5$; number of average $(\mathrm{NEX})=1$; the HRMRI scan time was 5 min and $28 \mathrm{sec}$.

\section{Imaging analysis}

The morphological evaluation of the plaque and the measurement of the vessels were performed on the custom-designed software of CASCADE (University of Washington, Seattle, Washington). Maximal Lumen Narrowing (MLN) was selected for lesion slice and the normal vessels at the proximal or distal end of the lesion were selected as the Referential Lumen ( $R L)$. The regions of interest (ROIs) were manually drawn to calculate the cross-sectional area of the vessel, including Lumen Area (LA), Vessel Area (VA), maximal Wall Thickness (WTmax), and minimal Wall Thickness (WTmin). The ROls for each measurement were drawn twice by two neuroradiologists who had more than 2 years of experience. The average of the measurements of the two neuroradiologists was obtained as the final result. The quantitative calculation of the pertinent parameters was performed as follows: (1) Degree of stenosis = $\left(1-\mathrm{LA}_{\mathrm{MLN}} / \mathrm{LA}_{\mathrm{RL}}\right) \times 100 \%$; (2) The $\mathrm{RI}=\mathrm{VA}_{\mathrm{MLN}} / \mathrm{VA} \mathrm{RL}_{\mathrm{RL}}, \mathrm{RI} \geq 1.05$ was defined as positive remodeling, $\mathrm{RI} \leq 0.95$ was defined as negative remodeling, RI between 0.95 and 1.05 was defined as non-remodeling. (3) Eccentricity degree $=\left(\mathrm{WT}_{\max }-\mathrm{WT} \mathrm{T}_{\min }\right) \times 100 \%$. The eccentric plaque was defined as eccentricity degree $\geq$ 0.5 , whereas the concentric plaque was defined as eccentricity $\mathbb{0} 0.5$.

\section{Statistical analysis}

All data were analyzed using SPSS 25.0 software. The quantitative data were expressed as mean \pm standard deviation. The categorical variables were summarized using counts and percentages. The Chisquare test was used to assess the differences in the categorical variables. The T-test and Wilcoxon's signed-rank tests were used for the analysis of the quantitative data. Logistic regression analysis was used to determine the differences in the plaques between the FTP and the control groups by adjusting for the confounding factors. A two-tailed $P$ value $\leq 0.05$ was considered to indicate a statistically significant difference.

\section{Results}

\section{Clinical characteristics of the study population}

A total of 58 patients (mean age: $60.76 \pm 10.11$ years, range: $31-80$ years; 35 males and 23 females) who met the inclusion criteria were finally enrolled and 12 patients were excluded due to lack of clinical information or absence of plaques on HRMRI imaging. The patients were classified as the FTP group $(n=$ $25)$ and the control group $(n=33)$. There were significant differences in diabetes between the two groups 
$(P<0.05)$, but no statistical significance in other variables, including age, sex, clinical risk factors of smoking, drinking, hypertension and hyperlipidemia (all $\mathrm{P}$ values $<0.05)($ Table 1$)$.

Table 1

Characteristics of the study patients $(n=58)$

\begin{tabular}{|llll|}
\hline & $\begin{array}{l}\text { FTP group } \\
(\mathbf{n = 2 5 )}\end{array}$ & $\begin{array}{l}\text { Control group } \\
(\mathbf{n = 3 3 )}\end{array}$ & P value \\
\hline Age, years & $62.96 \pm 7.987$ & $59.09 \pm 11.295$ & 0.151 \\
\hline Gender, male & $13(52 \%)$ & $22(66.7 \%)$ & 0.258 \\
\hline Smoking & $5(20 \%)$ & $11(33 \%)$ & 0.261 \\
\hline Drinking & $4(16 \%)$ & $7(21.2 \%)$ & 0.616 \\
\hline Hypertension & $18(72 \%)$ & $22(66.7 \%)$ & 0.664 \\
\hline Hyperlipidemia & $3(12 \%)$ & $2(6.1 \%)$ & 0.643 \\
\hline Diabetes & $14(56 \%)$ & $9(27.3 \%)$ & $0.027 *$ \\
\hline BMl & $25.383 \pm 3.708$ & $24.498 \pm 2.794$ & 0.700 \\
\hline * Statistically significant & & \\
\hline
\end{tabular}

\section{Differences in plaque characteristics of MCA and VBA between the two groups}

Based on the results of our study, plaques of the FTP group and the control group were mostly located in MCAs ( $50 \%$ vs. $53.7 \%$ ) and VBAs (45\% vs. $42.6 \%$ ) (Table 2). The results of the plaque characteristics in MCA and VBA between the FTP and the control groups are shown in Table 3. The FTP group indicated a slighter stenosis degree of MCAs (44\% vs. $60 \%, P=0.046)$ and higher remodeling index, and positive remodeling ( 1.095 vs. $0.875, P=0.004)$ compared with those of the control group. No significant differences were noted in the characteristics of the VBA plaques between the two groups $(P>0.05$ for all comparisons, as shown in Table 4). The representative images indicating the different plaque features in the two groups are shown in Figs. 1 and 2. 
Table 2

Distribution of plaques

\begin{tabular}{|lll|}
\hline & FTP group $(\mathbf{n}=\mathbf{4 0})$ & Control group $(\mathbf{n}=\mathbf{5 4})$ \\
\hline ACAs & $1(2.5 \%)$ & $1(1.9 \%)$ \\
\hline MCAs & $20(50 \%)$ & $29(53.7 \%)$ \\
\hline PCAs & $1(2.5 \%)$ & $1(1.9 \%)$ \\
\hline VBAs & $18(45 \%)$ & $23(42.6 \%)$ \\
\hline $\begin{array}{l}\text { ACA, anterior cerebral artery; MCA, middle cerebral artery; PCA, posterior cerebral artery; VBA, } \\
\text { vertebrobasilar artery. }\end{array}$ \\
\hline
\end{tabular}

Table 3

Morphological features of the MCA plaque

\begin{tabular}{|c|c|c|c|}
\hline & $\mathrm{n}(\%)$ or Mean \pm SD & & $\mathbf{P}$ \\
\hline & FTP group $(n=20)$ & Control group $(n=29)$ & value \\
\hline $\mathrm{LA}_{\mathrm{MLN}}\left(\mathrm{mm}^{2}\right)$ & $0.949 \pm 0.692$ & $0.820 \pm 0.471$ & 0.879 \\
\hline $\mathrm{VA}_{\mathrm{MLN}}\left(\mathrm{mm}^{2}\right)$ & $9.169 \pm 2.952$ & $9.133 \pm 3.052$ & 0.714 \\
\hline Degree of stenosis (\%) & $44 \pm 24$ & $60 \pm 21$ & $0.046^{\star}$ \\
\hline Remodeling Index & $1.095 \pm 0.248$ & $0.875 \pm 0.255$ & $0.004^{*}$ \\
\hline positive remodeling & $10(50 \%)$ & $7(24.1 \%)$ & \\
\hline negative remodeling & $3(15 \%)$ & $15(51.7 \%)$ & \\
\hline Eccentricity degree & $1.200 \pm 0.460$ & $1.135 \pm 0.476$ & 0.640 \\
\hline Eccentric plaque & $20(100 \%)$ & $26(90 \%)$ & \\
\hline Enhancement & $13(65 \%)$ & $23(79.3 \%)$ & 0.265 \\
\hline
\end{tabular}


Table 4

Morphological features of the VBA plaques

\begin{tabular}{|c|c|c|c|}
\hline & $\mathrm{n}(\%)$ or Mean \pm SD & & $P$ value \\
\hline & FTP group $(n=18)$ & Control group $(n=23)$ & \\
\hline $\mathrm{LA}_{\mathrm{MLN}}\left(\mathrm{mm}^{2}\right)$ & $1.955 \pm 1.999$ & $1.598 \pm 1.257$ & 0.723 \\
\hline $\mathrm{VA}_{\mathrm{MLN}}\left(\mathrm{mm}^{2}\right)$ & $15.101 \pm 5.080$ & $17.685 \pm 7.878$ & 0.211 \\
\hline Degree of stenosis (\%) & $40 \pm 18$ & $44 \pm 21$ & 0.600 \\
\hline Remodeling Index & $1.109 \pm 0.352$ & $0.991 \pm 0.258$ & 0.220 \\
\hline positive remodeling & $8(44.4 \%)$ & $8(34.8 \%)$ & \\
\hline negative remodeling & $7(38.9 \%)$ & $10(43.5 \%)$ & \\
\hline Eccentricity degree & $1.323 \pm 0.514$ & $1.358 \pm 0.645$ & 0.850 \\
\hline Eccentric plaque & $17(94.4 \%)$ & $22(95.7 \%)$ & \\
\hline Enhancement & $16(88.9 \%)$ & $19(82.6 \%)$ & 0.679 \\
\hline
\end{tabular}

$L A_{M L N}$, lumen area (maximal lumen narrowing); $L A_{R L}$, lumen area (referential area); VAMLN vessel area (maximal lumen narrowing); $\mathrm{VA}_{\mathrm{RL}}$, vessel area (referential area); * statistically significant.

Figure 1. Images of a 52-year-old male patient with FTP. (A)The TOF-MRA manifests stenosis of the M1 segment of the right MCA (arrowhead). The presence of FTP is shown (arrow). (B)The coronary T1weighted HR-MRI demonstrates an enhanced plaque in the M1 segment of the right MCA (arrow). (C)The transverse T1-weighted HR-MRI indicates the presence of eccentric plaque (arrow). (D)Measurement at the most narrowed site in the T1-weighted HR-MRI: The vessel area was $5.61 \mathrm{~mm}^{2}$ (dotted white circle) and the lumen area was $1.12 \mathrm{~mm}^{2}$ (solid white circle). The remodeling index was calculated as follows: Remodeling index $=1.17$ (positive remodeling). FTP: fetal-type posterior cerebral artery; TOF: time of flight; MCA: middle cerebral artery.

Figure 2. Images of a 53-year-old male patient without FTP. (A)DWI indicates an acute ischemic stroke in the distribution of the right MCA. (B)The TOF-MRA manifests severe stenosis of the M1 segment of the right MCA (arrow). (C)The transverse T1-weighted HR-MRI demonstrates a plaque in the M1 segment of the right MCA (arrow). (D)Measurement at the most narrowed site in the T1-weighted HR-MRI: The vessel area was $7.43 \mathrm{~mm}^{2}$ (dotted white circle) and the lumen area was $0.4 \mathrm{~mm}^{2}$ (solid white circle). The remodeling index was calculated as follows: Remodeling index $=0.53$ (negative remodeling). FTP: fetaltype posterior cerebral artery; TOF: time of flight; MCA: middle cerebral artery.

\section{Logistic regression analysis of MCA plaque characteristics between the two groups}


Univariate logistic regression analysis indicated that the remodeling index of MCA was associated with FTP $(\mathrm{OR}=0.088 ; 95 \% \mathrm{Cl}, 0.009-0.876 ; \mathrm{P}=0.038)$. Other clinical characteristics and HRMRI findings did not differ between both groups. Following multivariate regression analysis, the remodeling index of MCAs indicated significant differences between the FTP and the control groups $(\mathrm{OR}=0.022 ; 95 \% \mathrm{Cl}, 0.001-$ $0.474 ; P=0.015)($ Table 5).

Table 5

Univariate and multivariate logistic regression analyses

\begin{tabular}{|lllllll|}
\hline & \multicolumn{3}{l}{ Univariate regression } & & \multicolumn{3}{c|}{ Multivariate regression } \\
\hline & OR & $95 \% \mathrm{Cl}$ & P value & OR & $95 \% \mathrm{Cl}$ & P value \\
\hline Age, years & 1.062 & $0.987-1.144$ & 0.107 & 0.939 & $0.876-1.007$ & 0.076 \\
\hline Gender, male & 0.164 & $0.024-1.128$ & 0.066 & 0.554 & $0.142-2.163$ & 0.395 \\
\hline Hypertension & 0.215 & $0.029-1.589$ & 0.132 & - & - & - \\
\hline Diabetes & 2.608 & $0.495-13.763$ & 0.259 & - & - & - \\
\hline BMI & 0.948 & $0.725-1.241$ & 0.948 & 1.039 & $0.827-1.306$ & 0.741 \\
\hline Degree of stenosis & 0.459 & $0.103-2.042$ & 0.307 & - & - & - \\
\hline Remodeling Index & 0.088 & $0.009-0.876$ & $0.038^{*}$ & 0.022 & $0.001-0.474$ & $0.015^{*}$ \\
\hline * Statistically significant & & & & & \\
\hline
\end{tabular}

\section{Discussion}

According to a previous study, the formation of FTP is not related to congenital genetic factors and acquired neck activity [10]. Instead, it is mainly related to the changes noted in cerebral hemodynamics [11]. The internal carotid artery in the FTP supports both PCA and MCA. The additional presence of the occlusion of the tentorium cerebellum affects the anterior and posterior circulation of the leptomeningeal collateral circulation $[12,13]$. These hemodynamic changes play an important role in the development of atherosclerosis [14-17]. Hong et al. reported that the presence of the FTP could increase the compensatory blood flow of the internal carotid artery system and reduce the vertebrobasilar blood flow [18], resulting in vascular dilation and reduced blood flow rate in MCA. Lu et al. demonstrated that the low blood flow rate could decrease Wall Shear Stress (WSS). Low WSS was closely associated with the formation of atherosclerotic plaques [19]. Endothelial cell damage and lipid deposition are caused by high WSS due to the growth of the plaque. This promotes the development of atherosclerotic plaques and positive vessel remodeling. Positive remodeling is a crucial mechanism of atherosclerotic plaque formation and is more likely to cause acute ischemic stroke [20-22].

The present study demonstrated that the FTP group was more prone to exhibit positive remodeling and had a lower degree of stenosis than the control group in MCA, whereas no significant difference was 
noted in the VBA plaques between the two groups. The results of Lambert et al. were consistent with the results reported in the present study [23]. In contrast to this conclusion, certain inconsistencies were noted in the data presented in the current study and those of Yang's study. The latter supported the conclusion that patients with severe vertebral basilar intracranial stenosis and poor collateral circulation were more likely to develop positive remodeling, diffuse plaque distribution, and plaque enhancement. The difference may be due to the fact that Yang et al. included patients with posterior circulation stroke or TIA with $\geq 70 \%$ vertebral basilar intracranial stenosis [5]. By contrast, the present study included all patients with a vertebral basilar plaque and did not examine the stenosis rates of the patients in detail.

In addition, the majority of the stroke patients in the present study demonstrated enhanced plaques in MCA and VBA, which also verified that plaque enhancement was a marker of plaque instability and acute stroke $[24,25]$. The previous studies demonstrated that enhanced plaques were associated with vessel wall neovascularization and inflammation[26,27]. Our finding was similar to the result of Wang et al. that enhanced plaques in the symptomatic group were more frequent than that in the asymptomatic group[28].

The present study is one of the first studies that focus on the difference between anterior and posterior cerebral circulating artery plaques caused by FTP. By contrast, the majority of the previous studies focused on ischemic stroke as a result of changes occurring in the intracranial collateral circulation caused by FTP. Despite its novelty, the present study exhibits several limitations. Firstly, the sample size used was small and a single-center study design was used. Certain patients could not be included due to incomplete clinical data. Secondly, data processing was performed by an independent investigator and not by software, which may be subject to selective bias. Thirdly, due to low image resolution, the components of the intracranial arterial plaques were not studied.

\section{Conclusion}

The present study suggests that FTP promotes the development of atherosclerotic plaques and positive vessel remodeling in MCA and that it is not associated with VBA plaque formation. Further validation of the components of the intracranial atherosclerotic plaques is required in future studies.

\section{Abbreviations}

FTP: Fetal-type posterior cerebral artery; MCA: middle cerebral artery; VBA: Vertebrobasilar artery; HRMRI: High-resolution magnetic resonance imaging; VISTA: Volumetric isotropic turbo spin echo acquisition; LA: Lumen area; MLN: Maximal lumen narrowing; RI: Remodeling index; VA: Vessel area; WA: Wall area; WT: Wall thickness; ROI: Region of interest; WSS: Wall Shear Stress; TIA: Transient ischemic attack

\section{Declarations}

\section{Ethics approval and consent to participate}


The study protocol was approved by Zhongda Hospital Southeast University Ethics Committee. All participants signed written informed consent.

\section{Consent for publication}

Not applicable.

\section{Availability of data and materials}

The datasets generated and analyzed during the current study are used with the permission of Zhongda Hospital and are therefore not publicly available but are available from the corresponding author on reasonable request.

\section{Competing interests}

None.

\section{Funding}

The present study was supported by grants of Health and Family Planning Commission of Jiangsu Province (H2017008), Natural Science Foundation of Jiangsu Province (BK20170704).

\section{Authors' contributions}

LDQ conceived and designed the research and acquired the data. CXH provided the images. LDQ and XRJ analyzed and interpreted the data. LDQ draft the manuscript. ZDL and LC made critical revisions of the manuscript. All authors approved the final manuscript.

\section{Acknowledgements}

Not applicable.

\section{References}

1. Benjamin EJ, Muntner P, Alonso A, Bittencourt MS, Callaway CW, Carson AP, et al. Heart Disease and Stroke Statistics-2019 Update: A Report From the American Heart Association. Circulation. 2019;139 10.

2. Zou XD, Chung YC, Zhang L, Han Y, Yang Q, Jia J. Middle Cerebral Artery Atherosclerotic Plaques in Recent Small Subcortical Infarction: A Three-Dimensional High-resolution MR Study. Biomed Res Int. 2015;2015:540217; doi: 10.1155/2015/540217.

3. Qureshi Al, Caplan LR. Intracranial atherosclerosis. Lancet. 2014;383 9921:984-98; doi: 10.1016/S0140-6736(13)61088-0 \%/ Copyright (c) 2014 Elsevier Ltd. All rights reserved.

4. Xu WH, Li ML, Gao S, Ni J, Zhou LX, Yao M, et al. In vivo high-resolution MR imaging of symptomatic and asymptomatic middle cerebral artery atherosclerotic stenosis. Atherosclerosis. 2010;212 2:507- 
11; doi: 10.1016/j.atherosclerosis.2010.06.035\%/ Copyright (c) 2010 Elsevier Ireland Ltd. All rights reserved.

5. Ming Y, Ning M, Liping L, Anxin W, Jing J, Zhikai H, et al. Intracranial collaterals and arterial wall features in severe symptomatic vertebrobasilar stenosis. Neurological Research. 2020;42 8.

6. Krabbe-Hartkamp MJ, van der Grond J, de Leeuw FE, de Groot JC, Algra A, Hillen B, et al. Circle of Willis: morphologic variation on three-dimensional time-of-flight MR angiograms. Radiology. 1998;207 1:103-11; doi: 10.1148/radiology.207.1.9530305.

7. ALPERS BJ, BERRY RG, PADDISON RM. Anatomical studies of the circle of Willis in normal brain. AMA Arch Neurol Psychiatry. 1959;81 4:409-18; doi: 10.1001/archneurpsyc.1959.02340160007002.

8. De Silva KR, Silva TR, Gunasekera WS, Jayesekera RW. Variation in the origin of the posterior cerebral artery in adult Sri Lankans. Neurol India. 2009;57 1:46-9; doi: 10.4103/0028-3886.48821.

9. Saeki N, Rhoton AL. Microsurgical anatomy of the upper basilar artery and the posterior circle of Willis. Journal of neurosurgery. 1977;46 5.

10. Schomer DF, Marks MP, Steinberg GK, Johnstone IM, Boothroyd DB, Ross MR, et al. The anatomy of the posterior communicating artery as a risk factor for ischemic cerebral infarction. $\mathrm{N}$ Engl J Med. 1994;330 22:1565-70; doi: 10.1056/nejm199406023302204.

11. Van Overbeeke JJ, Hillen B, Tulleken CA. A comparative study of the circle of Willis in fetal and adult life. The configuration of the posterior bifurcation of the posterior communicating artery. $J$ Anat. 1991;176:45-54.

12. Brozici M, van der Zwan A, Hillen B. Anatomy and functionality of leptomeningeal anastomoses: a review. Stroke. 2003;34 11:2750-62; doi: 10.1161/01.Str.0000095791.85737.65.

13. van Raamt AF, Mali WPTM, van Laar PJ, van der Graaf Y. The Fetal Variant of the Circle of Willis and Its Influence on the Cerebral Collateral Circulation. Cerebrovascular Diseases. 2006;22 4.

14. Davies PF, Tripathi SC. Mechanical stress mechanisms and the cell. An endothelial paradigm. Circ Res. 1993;72 2:239-45; doi: 10.1161/01.res.72.2.239.

15. Weinbaum S, Chien S. Lipid transport aspects of atherogenesis. J Biomech Eng. 1993;115 4B:602-10; doi: 10.1115/1.2895547.

16. Fung YC, Liu SQ. Elementary mechanics of the endothelium of blood vessels. J Biomech Eng. 1993;115 1:1-12; doi: 10.1115/1.2895465.

17. Schmid-Schoenbein GW, Fung YC, Zweifach BW. Vascular endothelium-leukocyte interaction; sticking shear force in venules. Circ Res. 1975;36 1:173-84; doi: 10.1161/01.res.36.1.173.

18. Hong JM, Lee JS, Shin DH, Yong SW. Hemodynamic impact of fetal-variant Willisian circle on cerebral circulation: a duplex ultrasonography study. Eur Neurol. 2011;65 6:340-5; doi: 10.1159/000327213\%/ Copyright (c) 2011 S. Karger AG, Basel.

19. Sen L, Shusheng Z. Atherosclerosis research: the impact of physiological parameters on vascular wall stress. SN Applied Sciences. 2019;1 7. 
20. Zhang DF, Chen YC, Chen H, Zhang WD, Sun J, Mao CN, et al. A High-Resolution MRI Study of Relationship between Remodeling Patterns and Ischemic Stroke in Patients with Atherosclerotic Middle Cerebral Artery Stenosis. Front Aging Neurosci. 2017;9:140; doi: 10.3389/fnagi.2017.00140.

21. Shi, Wang, Zhou, Xing, Cheng, Feng, et al. Compensatory remodeling in symptomatic middle cerebral artery atherosclerotic stenosis: a high-resolution MRI and microemboli monitoring study. Neurological Research. 2012;34 2.

22. Wang Y, Bai Y, Wang ZX, et al. Evaluation of the relations between middle cerebral artery plaque characteristics and ischemic stroke and transient ischemic attack: three-dimensional high-resolution magnetic resonance imaging. Chin J Magn Reson Imaging, 2019, 10(3): 169-173.

23. Lambert SL, Williams FJ, Oganisyan ZZ, Branch LA, Mader EJ. Fetal-Type Variants of the Posterior Cerebral Artery and Concurrent Infarction in the Major Arterial Territories of the Cerebral Hemisphere. J Investig Med High Impact Case Rep. 2016;4 3:2324709616665409; doi:

$10.1177 / 2324709616665409$.

24. Wu F, Ma QF, Song HQ, Guo XH, Diniz MA, Song SS, et al. Differential Features of Culprit Intracranial Atherosclerotic Lesions: A Whole-Brain Vessel Wall Imaging Study in Patients With Acute Ischemic Stroke. Journal of the American Heart Association. 2018;7 15; doi: ARTN e009705 10.1161/JAHA.118.009705.

25. Lee HN, Ryu CW, Yun SJ. Vessel-Wall Magnetic Resonance Imaging of Intracranial Atherosclerotic Plaque and Ischemic Stroke: A Systematic Review and Meta-Analysis. Front Neurol. 2018;9:1032; doi: 10.3389/fneur.2018.01032.

26. Klein IF, Labreuche J, Lavallee PC, Mazighi M, Duyckaerts C, Hauw JJ, et al. Is Moderate Atherosclerotic Stenosis in the Middle Cerebral Artery a Cause of or a Coincidental Finding in Ischemic Stroke? Cerebrovascular Diseases. 2010;29 2:140-5; doi: 10.1159/000262310.

27. Kerwin WS, Oikawa M, Yuan C, Jarvik GP, Hatsukami TS. MR imaging of adventitial vasa vasorum in carotid atherosclerosis. Magn Reson Med. 2008;59 3:507-14; doi: 10.1002/mrm.21532.

28. Wang W, Yang Q, Li D, Fan Z, Bi X, Du X, et al. Incremental Value of Plaque Enhancement in Patients with Moderate or Severe Basilar Artery Stenosis: 3.0 T High-Resolution Magnetic Resonance Study. Biomed Res Int. 2017;2017:4281629; doi: 10.1155/2017/4281629.

\section{Figures}




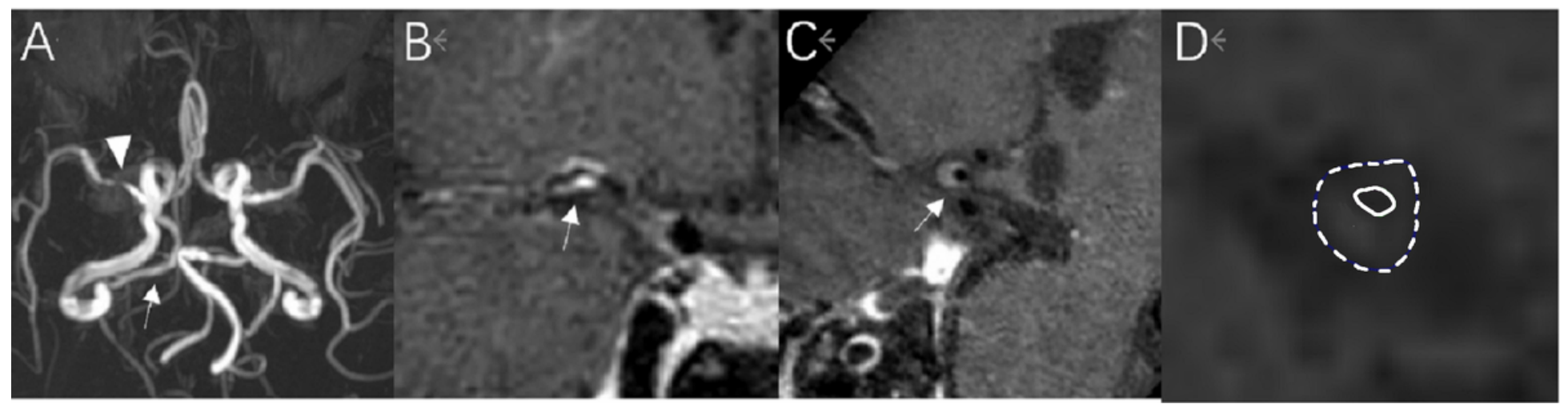

\section{Figure 1}

Images of a 52-year-old male patient with FTP. (A)The TOF-MRA manifests stenosis of the M1 segment of the right MCA (arrowhead). The presence of FTP is shown (arrow). (B)The coronary T1-weighted HRMRI demonstrates an enhanced plaque in the M1 segment of the right MCA (arrow). (C)The transverse T1-weighted HR-MRI indicates the presence of eccentric plaque (arrow). (D)Measurement at the most narrowed site in the T1-weighted HR-MRI: The vessel area was $5.61 \mathrm{~mm}^{2}$ (dotted white circle) and the lumen area was $1.12 \mathrm{~mm}^{2}$ (solid white circle). The remodeling index was calculated as follows: Remodeling index $=1.17$ (positive remodeling). FTP: fetal-type posterior cerebral artery; TOF: time of flight; MCA: middle cerebral artery.

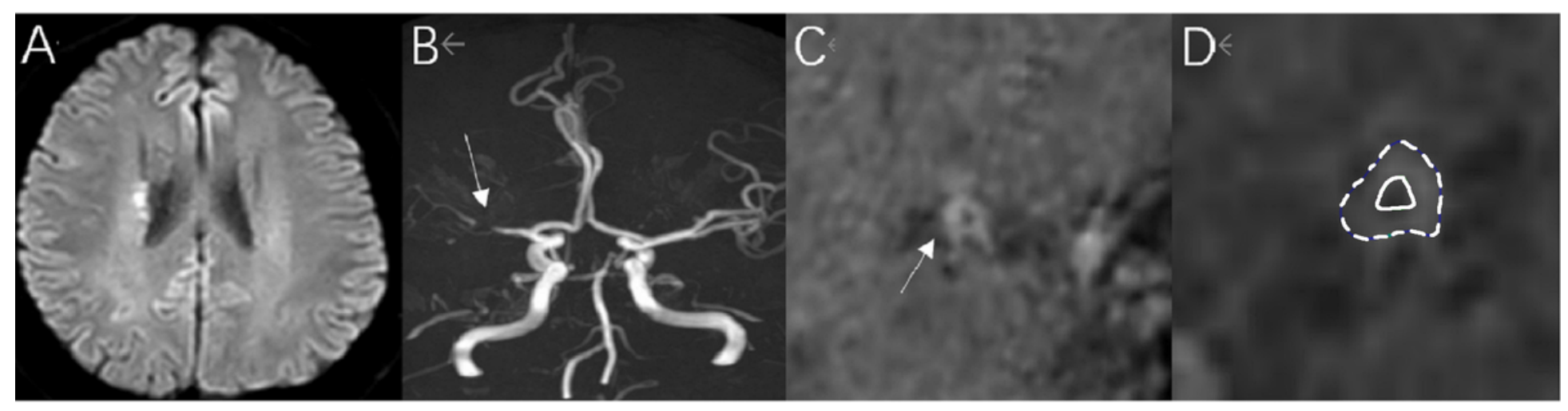

\section{Figure 2}

Images of a 53-year-old male patient without FTP. (A)DWI indicates an acute ischemic stroke in the distribution of the right MCA. (B)The TOF-MRA manifests severe stenosis of the M1 segment of the right MCA (arrow). (C)The transverse T1-weighted HR-MRI demonstrates a plaque in the M1 segment of the right MCA (arrow). (D)Measurement at the most narrowed site in the T1-weighted HR-MRI: The vessel area was $7.43 \mathrm{~mm}^{2}$ (dotted white circle) and the lumen area was $0.4 \mathrm{~mm}^{2}$ (solid white circle). The remodeling index was calculated as follows: Remodeling index $=0.53$ (negative remodeling). FTP: fetaltype posterior cerebral artery; TOF: time of flight; MCA: middle cerebral artery. 